\title{
Effects of compensating the temperature coefficient of frequency with the acoustic reflector layers on the overall performance of solidly mounted resonators
}

\author{
Junaid Munir , Teona Mirea , Mario DeMiguel-Ramos , M.A. Saeed ,Amiruddin Bin Shaari , \\ Enrique Iborra
}

Keywords:

Solidly mounted resonators

Temperature coefficient of frequency

Acoustic reflectors

Finite Element Analysis

\begin{abstract}
A B S T R A C T
Thin film acoustic wave resonator based devices require compensation of temperature coefficient of frequency (TCF) in many applications. This work presents the design and fabrication of temperature compensated solidly mounted resonators (SMRs). The characteristics of each material of the layered structure have an effect on the device TCF but depending on the relative position with respect to the piezoelectric material in the stack. The influence of material properties of the different layers composing the device on the TCF is discussed in detail. TCF behavior simulation is done with Mason's model and, to take into account the deterioration of overall performance due to the finite lateral size and shape of the resonator, we have used 2D and 3D finite element modelling of the resonators. The overall behavior of the device for external loads is predicted. SMRs are designed according to simulations and fabricated with different configurations to obtain TCF as near to zero as possible with an optimized response. Resonators are made by depositing Mo/AIN/Mo piezoelectric stacks on acoustic reflectors. As reflector materials, conductive $\mathrm{W}$ and insulating $\mathrm{WO}_{\mathrm{x}}$ films have been used as high acoustic impedance materials. $\mathrm{SiO}_{2}$ films are used as low acoustic impedance material.
\end{abstract}

\section{Introduction}

Bulk acoustic wave resonators (BAW) based sensors are gaining much attention for chemical and physical sensing applications, because of their simple and robust structure, interconnection with electrical systems, the possibility of low-cost mass production and high sensitivity $[1,2]$. Their potential application in mobile communication, as competitors of surface acoustic wave (SAW) and dielectric filters, and in biological and gas sensing, are widely reported [3-5]. The thickness-excited longitudinal wave mode in bulk acoustic wave resonators (both suspended and solidly mounted (SMR)) has been used in gravimetric chemical and physical sensors working in gas phase and they show encouraging results for bio and smart gas sensor applications [6,7]. Some attempts $[8,9]$ have been made to operate these resonators for biochemical sensing handling liquid samples. FBAR based on $c$-axisinclined $\mathrm{ZnO}[10]$ and AIN [9] operating in the thickness shear mode have been successfully used in liquid media, indicating a great potential for chemical and biochemical sensors as high sensitivity transducers.

Temperature stability in FBAR and SMRs for sensor applications is another non-resolved critical issue. The thermal stability of the resonator can be evaluated by the temperature coefficient of the resonant (or anti-resonant) frequency (TCF), which measures the relative variations of resonant frequency with temperature $(\mathrm{TCF}=(1 / f) \cdot(d f / d T))$. Since there is no piezoelectric material available which is temperature compensated, it is compulsory to use composite structures in such a way that some materials compensate the negative coefficients of the common piezoelectric materials. The variation of the resonant frequency with temperature can be due to the variation of material properties such the acoustic velocity, which depends on the elastic coefficients, and the variation of the geometry due to dilatation processes. Reducing TCF in a resonator is usually achieved by adding films which have properties that compensate the rifts of those that move with temperature. The most common material used for this purpose is $\mathrm{SiO}_{2}$, which has a positive coefficient of the elastic constants and compensates the 
negative one of the other materials composing the resonator [11]. $\mathrm{ZnO}$ and $\mathrm{AlN}$ are both sensitive to temperature variation having a negative coefficient. The typical temperature coefficient of frequency (TCF) of suspended $\mathrm{ZnO}$ and AIN SMRs are $-60 \mathrm{ppm} /{ }^{\circ} \mathrm{C}$ and $-25 \mathrm{ppm} /{ }^{\circ} \mathrm{C}$ respectively [12]. To compensate this negative TCF, an additional layer of material having positive TCF in the acoustic path is needed. Successfully compensation of thickness shear mode by [13] in $\mathrm{ZnO} / \mathrm{Si}$ and AlN/Si composite FBARs has been reported. In this case, Si was $\mathrm{p}^{+}$doped to achieve a positive TCF, but it results in a significant reduction of both electromechanical coupling coefficient $\left(k_{t}^{2}\right)$ and resonant frequency. The TCF of AlN is reduced to $-15 \mathrm{ppm} /{ }^{\circ} \mathrm{C}$ [14] by using $\mathrm{SiO}_{2}$ films (which display a TCF of $+85 \mathrm{ppm} /{ }^{\circ} \mathrm{C}$ ) as a compensating material. The added material also results in a substantial reduction of coupling and resonant frequency.

This paper reports the simulation at different temperatures and the electrical characterization of fabricated SMRs using $\mathrm{SiO}_{2} / \mathrm{W}$ and $\mathrm{SiO}_{2} / \mathrm{WO}_{\mathrm{x}}$ reflectors. Different kinds of the reflector are simulated and fabricated with different thickness from the ideal symmetric $\lambda / 4$ to asymmetric with AlN as a piezoelectric material. We report the influence of the different thicknesses of the layers composing the acoustic reflectors on the TCF. All the simulations are done with Mason model and Finite Element Modelling (FEM) through acoustic velocity dependence on temperature and thermal expansion respectively. It is worth to note that in this work, $\mathrm{SiO}_{2}$ layers are part of the reflector. This preserves the performance of the resonators (coupling and quality factor).

\section{Simulation methods}

The one dimensional simulation method based on Mason's model [15] is proposed for determining the influence of different materials and their thicknesses in the reflector stack on the TCF of SMRs. The accurate design of the resonator is needed before fabrication. Mason's model provides a good tool for driving the resonant frequencies of the different modes at a time. It can also be used as designing tool to determine the thicknesses of the different layers of the acoustic reflector to minimize the TCF and their influence on the performance of the resonator.

The energies associated with the transmitted and reflected wave can be easily calculated, if we consider that a wave is propagating from the piezoelectric layer towards the sandwiched reflector layers. The transmitted and reflected wave energy can be computed with the help of the effective acoustic impedance $Z_{b}$ of the bottom layer stack of Mason's model. For this purpose, the mirror reflectivity is given by

$R=\frac{Z_{p}-Z_{b}}{Z_{p}+Z_{b}}$

where $Z_{p}$ and $Z_{b}$ is the acoustic impedance of the piezoelectric layer and the effective acoustic impedance of the layer stack underneath it respectively. $R, Z_{\mathrm{p}}$ and $Z_{\mathrm{b}}$ are complex numbers, which also take into account acoustic losses. In order to study the in band reflectivity, it is more convenient to use the inverse of $\mathrm{R}$ (transmittance $T$ ).

The reflector transmittance is affected when the typical symmetric configuration (all layers of a $\lambda / 4$ thickness) are changed to asymmetric (some layers with a thickness different to $\lambda / 4$ ). Variation in the thickness of any one of the layers of the reflector below the AlN layer leads to deformation in the reflector $T$. This causes the variation of the leakages of energy from the piezoelectric stack and the possible degradation of overall performance. Increasing the thickness of $\mathrm{SiO}_{2}$ (top reflector layer) causes the shear and longitudinal resonances into this layer to appear in the transmission band of the reflector. The deformation generated in this layer is transmitted to the AlN film causing a piezoelectric field that is measured as impedance variations as shown in Fig. 1. For simulating the transmittance spectrum of the different reflectors in Fig. 1, material constants are taken from [16-18].

Fig. 1 shows that increasing the uppermost $\mathrm{SiO}_{2}$ layer thickness of the reflector distorts its transmittance and inaugurates overtone modes in the electrical spectrum at frequencies in the band of interest and causes to decrease the acoustic reflectance at major frequencies. It can be seen that further increase in reflector uppermost layer thickness leads to higher distortion in the reflectance. The additional energy loss due to the reduction of the acoustic reflectance can be reduced by carefully designing the whole layer stack and a maximum reflector response can be achieved at the desired frequencies as shown in Fig. 1. It is also notable that this increase of the $\mathrm{SiO}_{2}$ thickness also causes the decrease of the resonant frequencies generated in the AIN film as the effective mass of the whole resonator varies (gravimetric effect). This, of course, can be corrected by redefining the thickness of the AlN layer. If the increase of the thickness of the uppermost $\mathrm{SiO}_{2}$ layer is high enough, the frequencies of the resonances in it, the overtones, appear at lower values than those corresponding to the main resonances (shear and longitudinal) generated in the AlN film. This situation is observed in Fig. 1(d). To identify the origin of these resonances is not straightforward and only by measuring their TCF one can definitively make this identification. TCF of the modes originated in $\mathrm{SiO}_{2}$ are much more positive than those generated in AlN. All these behaviors are present not only in simulation, but also in experimental results. Maximum reflectivity of the acoustic reflector at the resonant frequency is essential because radiation of acoustic waves to the substrate represents energy losses that decrease the quality factor of the device [19]. To maximize reflectivity, it is essential to increase the acoustic impedance ratio between the acoustic layered materials, but also to modify the thickness of all layers when the uppermost $\mathrm{SiO}_{2}$ layer is made thicker for TCF compensation. The design of the piezoelectric stack (AlN and electrodes) has also to take into account all these issues. Mason's model is a very efficient tool to do this. However, this is a one-dimensional model and no lateral effects are taken into account. To do it on the overall performance of the stack, we use precise $2 \mathrm{D}$ and $3 \mathrm{D}$ numerical simulation through Finite Element Analysis using COMSOL Multiphysics software. In 2D and 3D models, the finite element method in COMSOL software is used to solve the interaction between mechanical displacement and electrical potential by employing following constitutive piezoelectric equations [20].

$\mathrm{T}_{\mathrm{i}}=\mathrm{c}_{\mathrm{ij}}^{\mathrm{E}} \mathrm{S}_{\mathrm{j}}-\mathrm{e}_{\mathrm{ij}} \mathrm{E}_{\mathrm{j}}$

$D_{i}=\varepsilon_{i j}^{s} E_{j}-e_{i j} S_{j}$

where $T_{i}$ and $S_{j}$ are the stress and strain components. $C_{\mathrm{ij}}$ is the stiffness constant, $e_{\mathrm{ij}}$ is the piezoelectric stress constants and, $E_{\mathrm{j}}$ is the electrical field component. $\varepsilon_{\mathrm{ij}}$ is the permittivity constants and $D_{\mathrm{i}}$ the electrical displacement field. The superscripts $\mathrm{S}$ and $\mathrm{E}$ indicate that the respective constants are measured at a constant strain and electric field respectively. The frequency response of the resonators and the displacement profile of the wave in the resonator stack are evaluated with 2D FEM. Perfectly matched layers (PLMs) are used at the boundary edges of the stack to avoid energy leakage due to the reflection of waves from the boundaries. The bottom electrode is set to ground, and the frequency dependent potential of $1.0 \mathrm{~V}$ is applied to the top electrode. At the boundaries of the PMLs domain, the fixed constraint condition is applied. The other layers are set as free. The mashing is done with mapped mash having several distributions.

The displacement profile of longitudinal waves of the asymmetric resonator (W reflectors) with $1.21 \mu \mathrm{m}$ thick layer of $\mathrm{SiO}_{2}$ at the resonant frequency is simulated with $2 \mathrm{D}$ finite element model as 

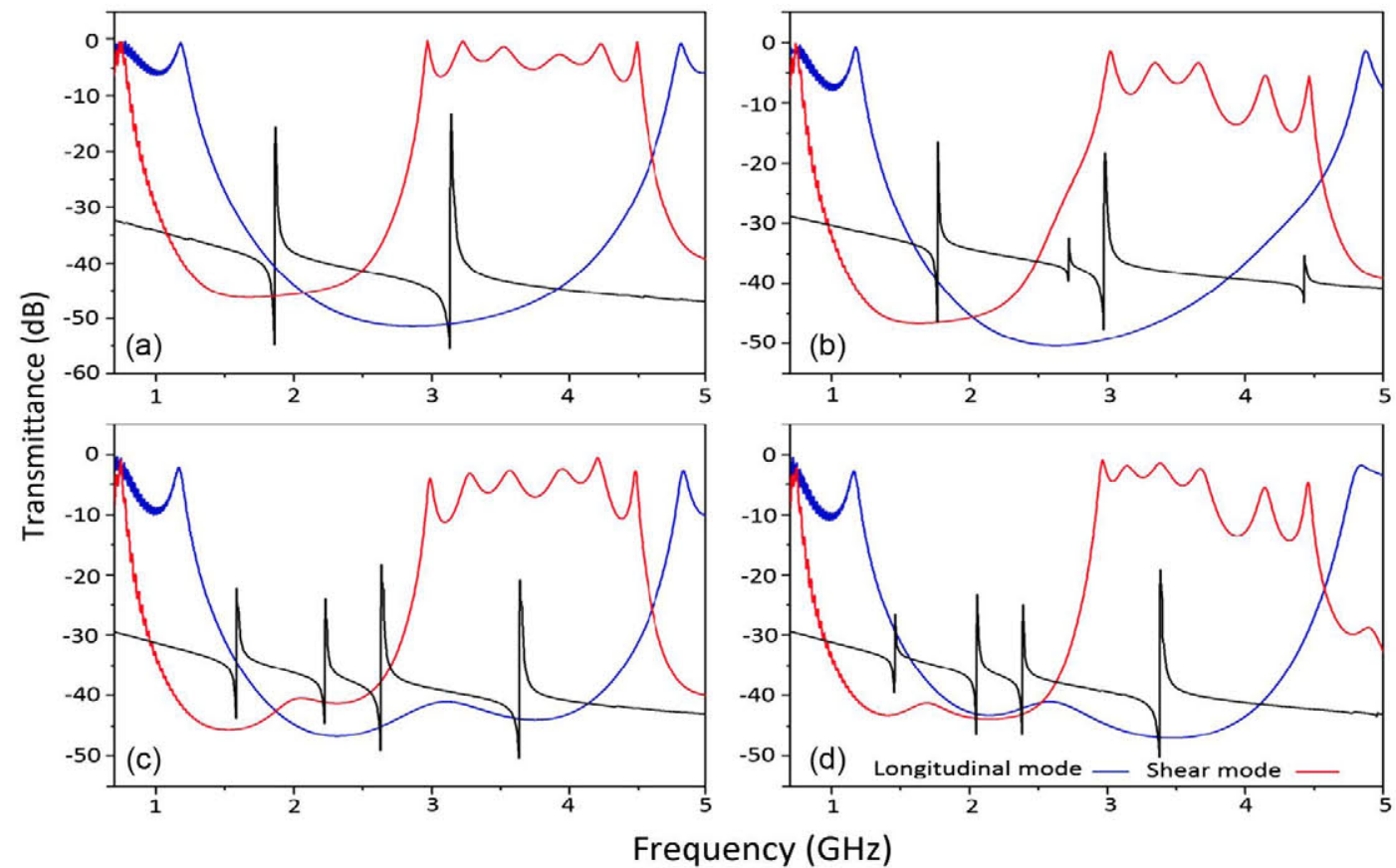

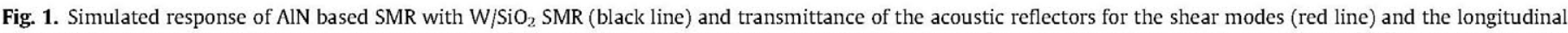

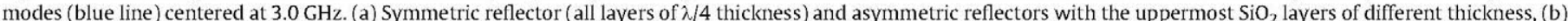

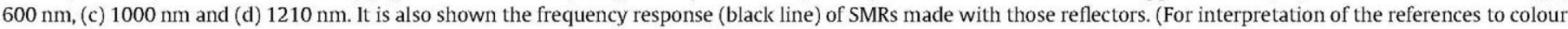
in this figure legend, the reader is referred to the web version of this article.)

shown in Fig. 2. It can be seen that no significant energy is radiated to the substrate or edges, and all energy is confined inside the resonator, which confirms a good performance of the reflectors in the stack. However, further increase causes the energy to leak into the substrate.

Although 2D FEM provides a good approach to evaluating the frequency response of the SMRs, a 3D FEM model has also been developed to calculate the overall response of the resonator. In this model, symmetric boundary conditions are applied to the relevant boundaries. Similar kind of boundary and electric conditions are applied as in 2D model. To reduce the computational time, only a quarter of the original structure is simulated with 3D geometry and for appropriate boundaries, symmetric conditions are applied. The ground and electric conditions are employed in the same way as in 2D geometry. The spurious response of the SMRs is also calculated by the 3D model. As very thin layers are used in a 3D model, a swept mesh and triangular mesh elements are used to mesh the entire model. The displacement profile is same as obtained with 2D model. The following expression is used to simulate the electrical impedance using the pzd interface.

$$
Z=\frac{V}{j \omega Q}
$$

where $Z, V, \omega$ and $Q$ represent the electrical impedance, applied voltage, angular frequency and the charge on the top electrode surface, respectively. The stress profile and impedance simulated with CONSOL Multiphysics is shown in Fig. 3. (a)

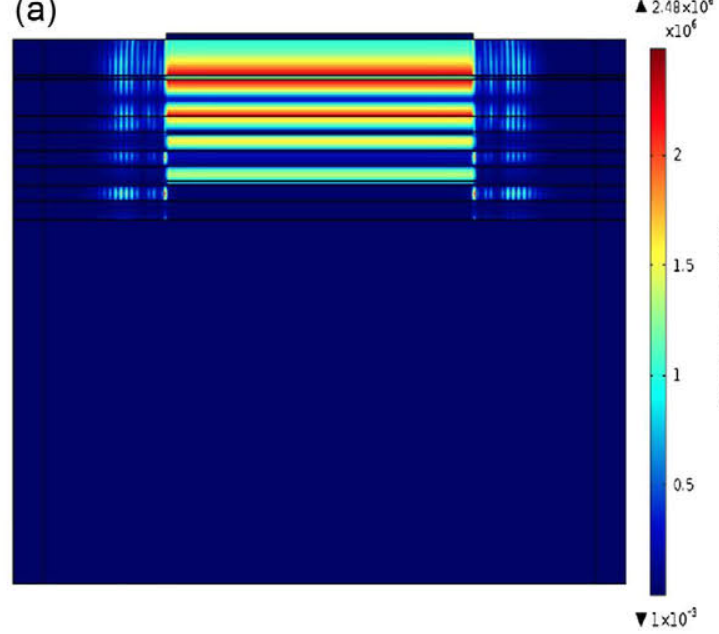

(b)

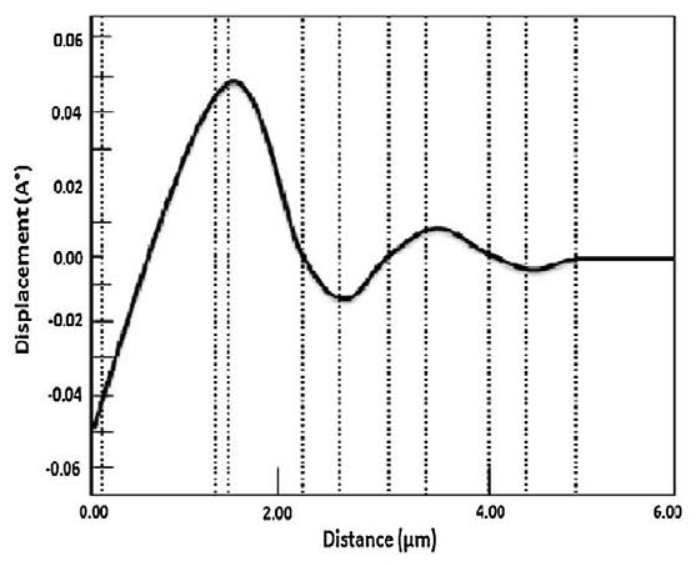

Fig. 2. (a) Displacement profile at the resonant frequency, (b) amplitude of standing wave as a function of depth for asymmetric acoustic reflectors with $2 \mathrm{D}$ finite element model. 


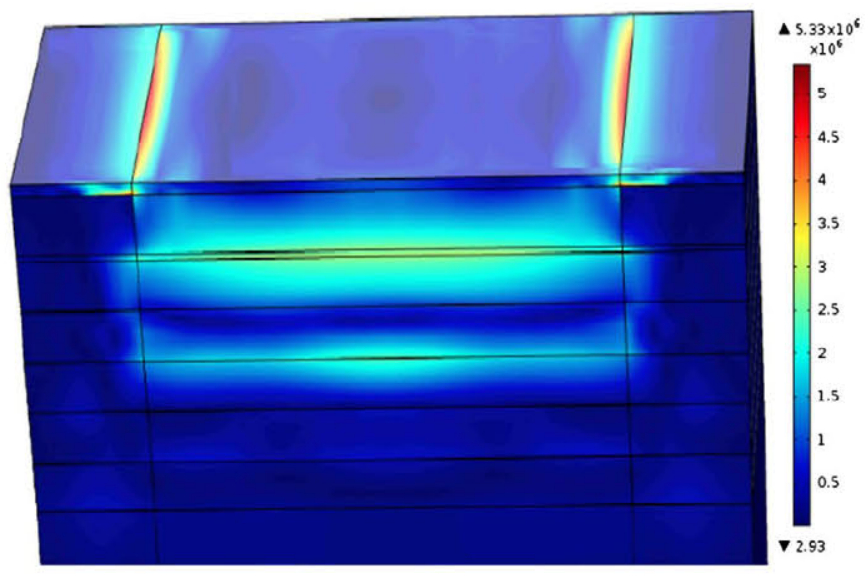

(a)

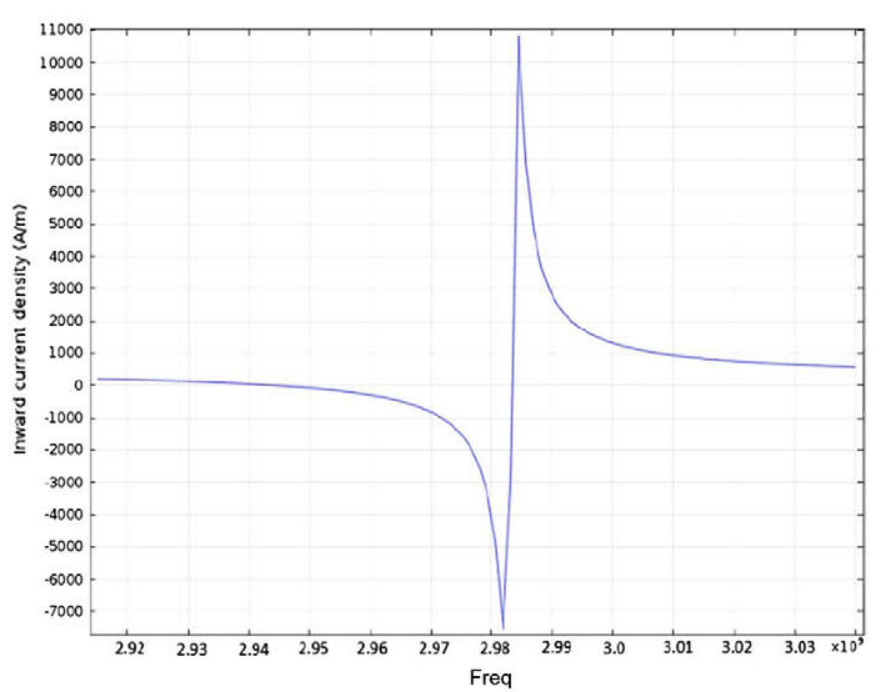

(b)

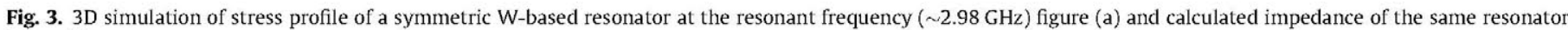
figure (b).

It can be seen from the stress profile that most stressful region is the bottom area of the piezoelectric layer and the top region of the first $\mathrm{SiO}_{2}$ layer. Fig. 3(b) shows the impedance spectrum calculated with 3-D finite element model and it is the same as the one calculated with Mason's model.

\section{Experimental}

Test devices for measuring the TCF and the influence of the reflector design on it were fabricated by depositing the piezoelectric layer sandwiched between the electrodes on top of the acoustic reflector. The bottom electrode consisted in $100 \mathrm{~nm}$ thick sputtered Mo film and the top one in $150 \mathrm{~nm}$ thick Mo layer. The thickness of the AlN was always $1000 \mathrm{~nm}$. Although this stack is not optimum for the different used reflectors, we used it in all the experiments to compare the influence of the thickness of the uppermost $\mathrm{SiO}_{2}$ layer of the reflector in the values of TCF. Acoustic reflectors with different configurations made of alternating layers of low $\left(\mathrm{SiO}_{2}\right)$ and high ( $\mathrm{W}$ or $\mathrm{WO}_{\mathrm{x}}$ ) acoustic impedance materials have been used. As substrate, $(100)$ oriented Si wafers $100 \mathrm{~mm}$ in diameter were used. AlN films were sputtered in an ultrahigh-vacuum system pumped to a base pressure below $1.3 \times 10^{-6} \mathrm{~Pa}$. A high purity aluminum target $150 \mathrm{~mm}$ in diameter was sputtered in $\mathrm{Ar} / \mathrm{N}_{2}$ atmospheres (40:60) using a pulsed-DC source (MKS ENI RPG-50E) working at $250 \mathrm{kHz}$. To study shear and longitudinal modes, tilted $\mathrm{c}$-axis and pure c-axis oriented AlN films were used. Pure c-axis oriented films were sputtered at low pressure $(0.27 \mathrm{~Pa})$ and $1.2 \mathrm{~kW}$ power. During deposition the substrate temperature was kept at $400^{\circ} \mathrm{C}$ and for biasing the substrate to a DC voltage of $-60 \mathrm{~V}$, an RF power of $15 \mathrm{~W}$ was used. This substrate biasing is intended to adjust the in plane residual stress to values lower than $200 \mathrm{MPa}$. This stress is important to be maintained as low as possible because it can generate delamination of the structure when temperature changes. Under these conditions, the rate of film growing at the center of the substrate was $45 \mathrm{~nm} / \mathrm{min}$. To deposit AlN films with tilted grains, before the main AlN film deposited under the same conditions, an AlN seed layer was deposited at high pressure ( $0.66 \mathrm{~Pa})$ and $600 \mathrm{~W}$ without deliberately biasing or heating the substrate. The seed layers of AlN exhibit a (1.03)-preferred orientation, which is crucial to stimulate the growth of the consequent AlN tilted films provided by these conditions [21]. Additionally, directional deposition of the active piezoelectric layer is needed, therefore the samples were located at $4 \mathrm{~cm}$ from the center of the substrate holder and only $34 \mathrm{~nm} /$ min of deposition rate was achieved. Using these conditions, AlN films with the wurtzite c-axis tilted around $24^{\circ}$ with respect to the surface normal are obtained. The Mo bottom electrode is continuous while the top one is dry-etching patterned. The geometry of the resonator consist in a top electrode surrounding by an extend ground plane in a ground-signal-ground (GSG) configuration. Ground plane contacts the bottom electrode by a strong capacitive coupling.

The acoustic reflectors comprised three layers of high acoustic impedance material ( $\mathrm{W}$ or $\mathrm{WO}_{\mathrm{x}}$ ) alternated with four layers of low acoustic impedance material (dense $\mathrm{SiO}_{2}$ ). Pulsed-DC sputtering of $150 \mathrm{~mm}$ targets in a Leybold Z550 system was used to deposit all layers. To get uniform thickness across the $100 \mathrm{~mm}$ silicon wafer, a system of screens between the rotating substrate and the targets was used. $\mathrm{Si}$ target was used to deposit $\mathrm{SiO}_{2}$ layers in $\mathrm{Ar} / \mathrm{O}_{2}$ mixtures with low pressure to ensure a dense material with positive temperature coefficient of the acoustic velocity. The sputtering of high impedance layers $\left(\mathrm{W}\right.$ and $\mathrm{WO}_{\mathrm{x}}$ ) was done at low pressure $(0.13 \mathrm{~Pa}) .100 \mu \mathrm{m}$ pitch $\mathrm{RF}$-probes Picoprobe from GGB Industries with an Agilent N5230A network analyzer was used for on wafer measuring the electrical impedance of the SMRs as a function of the frequency and temperature. We use segmented frequency scans to acquire 4000 samples around each resonance for accurately determining the resonant and antiresonant frequencies. During measurements, the sample temperature was varied from $25^{\circ} \mathrm{C}$ to $100^{\circ} \mathrm{C}$ in steps of around $10^{\circ} \mathrm{C}$ by a heater in the sample holder and a temperature controller. A type-K thermocouple was used to measure the temperature of the surface of the sample by attaching it with a high thermally conductive.

\section{Results and discussion}

In the present work, a theoretical model for the temperature compensated solidly mounted resonator is presented. We start with a symmetric configuration (i.e. $\lambda / 4$-thickness reflector) and we continue with the asymmetric configuration in which the thickness of top two reflector layers is varied to observe its effects on the TCF of SMRs. With the increase in temperature, there is a 
decrease in the stiffness constant of most of the materials. It reduces the acoustic velocity, which leads to the negative TCF. However, $\mathrm{SiO}_{2}$ [22] has a positive temperature coefficient of its elastic constants, which results in an increase of the acoustic velocity with temperature, thus showing a positive TCF. The use of $\mathrm{SiO}_{2}$ as low impedance material in acoustic reflectors helps to compensate the TCF of the negative temperature coefficient of other materials. With the increase in temperature, the acoustic velocities decrease and as a result, the resonant frequency shifts to lower values. This can be partially compensated by increasing the thickness of $\mathrm{SiO}_{2}$ layer. To observe this effect, thermal expansion is applied in COMSOL Multiphysics software using the following expression.

$\varepsilon_{\text {inel }}=\alpha\left(T-T_{\text {ref }}\right)$

where $\varepsilon_{\text {inel }}$ is the fractional change in length by thermal expansion, $\alpha$ is the coefficient of thermal expansion, $T$ and $T_{\text {ref }}$ are the actual and reference temperatures respectively. However, the major contributing source to the TCF is the thermal variation in the elastic constant of materials, which directly affects their acoustic velocities. From COMSOL simulations, it is observed that other thermal effects such as thermal expansion can also affect the TCF as in Fig. 4. It shows a decrease in the resonant frequency with every $20^{\circ} \mathrm{C}$ rise in temperature.

An increase in $\mathrm{SiO}_{2}$ thickness loads the resonator, which not only reduces the resonance frequency but also $k_{e f f}^{2}$ and $\mathrm{Q}$ values. A careful design of the whole stack helps to recover the frequency values and also to avoid the degradation due to energy loss.

Fig. 5 displays the normalized resonant frequency as a function of temperature for shear and longitudinal modes. Asymmetric Wbased acoustic reflectors are used in this configuration with AlN as a piezoelectric material with a non- $\lambda / 4$-thickness of $\mathrm{SiO}_{2}$ of $1.21 \mu \mathrm{m}$.

Measured TCF values are $-15.2 \mathrm{ppm} /{ }^{\circ} \mathrm{C}$ and $-6.6 \mathrm{ppm} /{ }^{\circ} \mathrm{C}$ for the shear and longitudinal wave modes of $\mathrm{AlN}$ and $+3.4 \mathrm{ppm} /{ }^{\circ} \mathrm{C}$ and $+23.2 \mathrm{ppm} /{ }^{\circ} \mathrm{C}$ for the shear and longitudinal overtones wave modes of $\mathrm{SiO}_{2}$ layers.

The TCF variations of both shear and longitudinal wave mode of the resonator made on $\mathrm{SiO}_{2}-\mathrm{W}$ reflectors are achieved by increasing the thickness of the $\mathrm{SiO}_{2}$ layer as shown in Fig. 6 .

The negative values of the TCF decrease when the thickness of the uppermost low impedance $\mathrm{SiO}_{2}$ layer increases. However, the values of the quality factor $\mathrm{Q}$ and electromechanical coupling

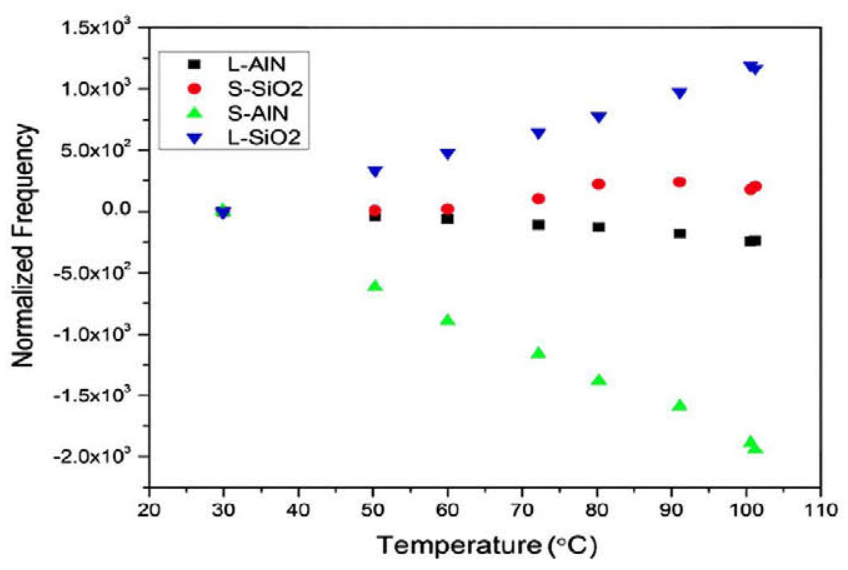

Fig. 5. Measured values of normalized resonant frequency vs. temperature of shear and longitudinal wave modes with asymmetric W-reflectors.

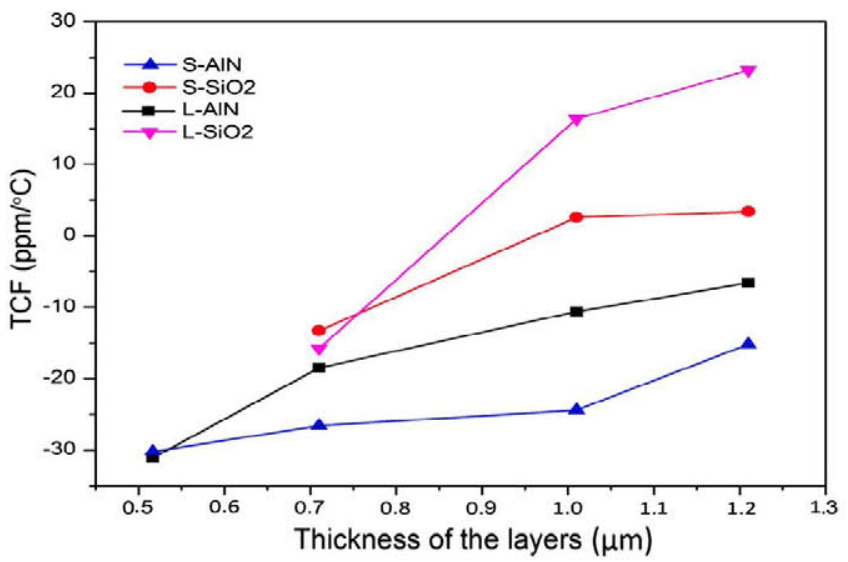

Fig. 6. Measured TCF values of the main ( $\mathrm{AlN})$ and secondary $\left(\mathrm{SiO}_{2}\right)$, shear $(\mathrm{S})$ and longitudinal ( $\mathrm{L}$ ) modes for asymmetric resonators as a function of the thickness of $\mathrm{SiO}_{2}$ in SMR reflectors.

coefficient $k_{\text {eff }}^{2}$ decrease simultaneously. The relation of the $Q$ factor and the $k_{\text {eff }}^{2}$ as a function of thickness of the layers in the $\mathrm{W}-\mathrm{SiO}_{2}$ based resonator is shown in Fig. 7.

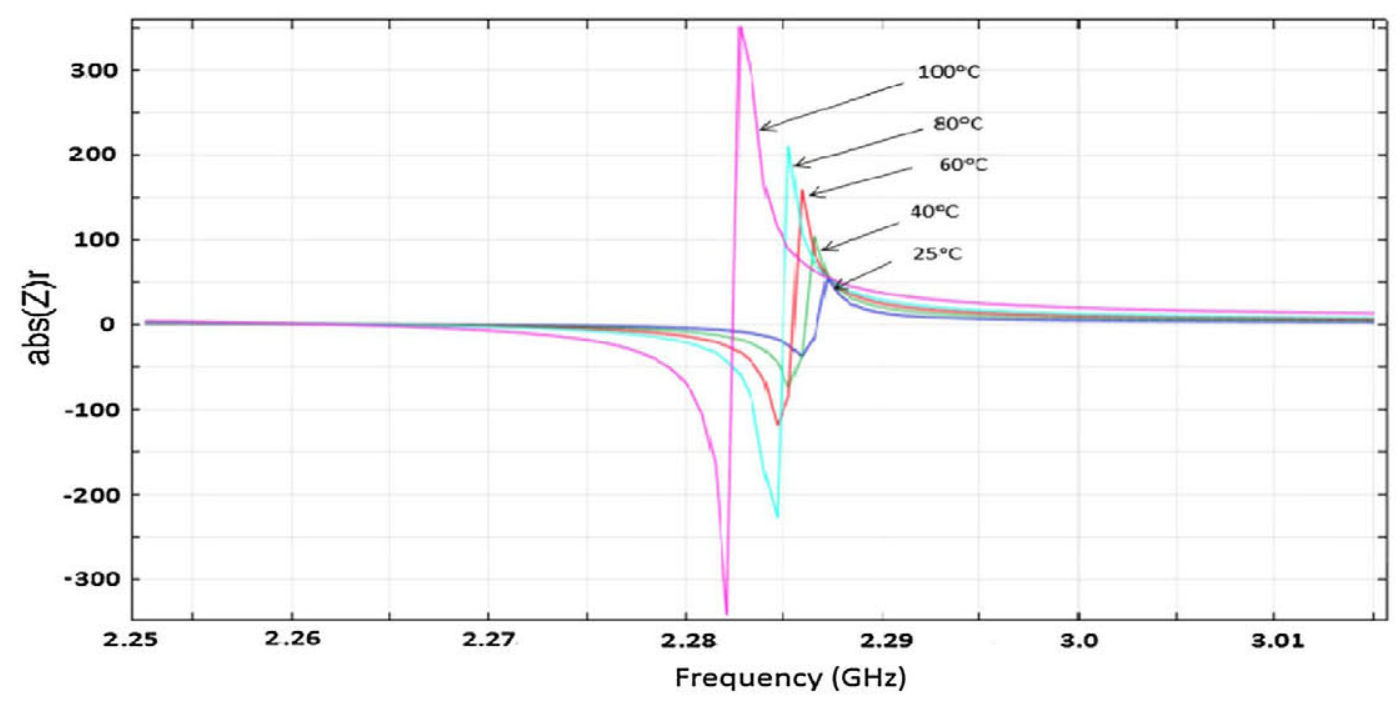

Fig. 4. Simulated spectra of a resonator at different temperatures with Finite Element Modelling (FEM). 
It can be seen that there is a constant decrease in the values of both $\mathrm{Q}$ and $k_{\text {eff }}^{2}$ with the increase of $\mathrm{SiO}_{2}$ thickness. Above $1 \mu \mathrm{m}$ thick $\mathrm{SiO}_{2}$, there is a sudden decrease in both $\mathrm{Q}$ and $k_{\text {eff }}^{2}$ values. At this thickness of $\mathrm{SiO}_{2}$, the TCF for longitudinal and shear modes of $\mathrm{SiO}_{2}$ is also positive. Further increase in $\mathrm{SiO}_{2}$ thickness compensates TCF but at a huge cost for the $\mathrm{Q}$ and $k_{\text {eff }}^{2}$ values.

A similar behavior was found in resonators made of insulating $\mathrm{WO}_{\mathrm{x}} / \mathrm{SiO}_{2}$ reflectors. It is important to note that a redesign of the piezoelectric capacitor, which do not do in this work for only change a variable and making the comparison easy, can improve the values of both $\mathrm{Q}$ and $k_{\text {eff }}^{2}$ values and recover the resonant frequency values. The data of the TCF of the $\mathrm{W} / \mathrm{SiO}_{2}$ and $\mathrm{WO}_{\mathrm{x}} / \mathrm{SiO}_{2}$ based symmetric and asymmetric reflectors with resonators operating in the shear and the longitudinal mode while varying the thicknesses of the corresponding uppermost layer $\left(\mathrm{SiO}_{2}, \mathrm{~W}\right.$ or $\mathrm{WO}_{\mathrm{x}}$ ) are given in Table 1.

There are only two, AlN shear and longitudinal, modes in symmetric reflectors. An increase in the thickness of the $\mathrm{SiO}_{2}$ layer leads

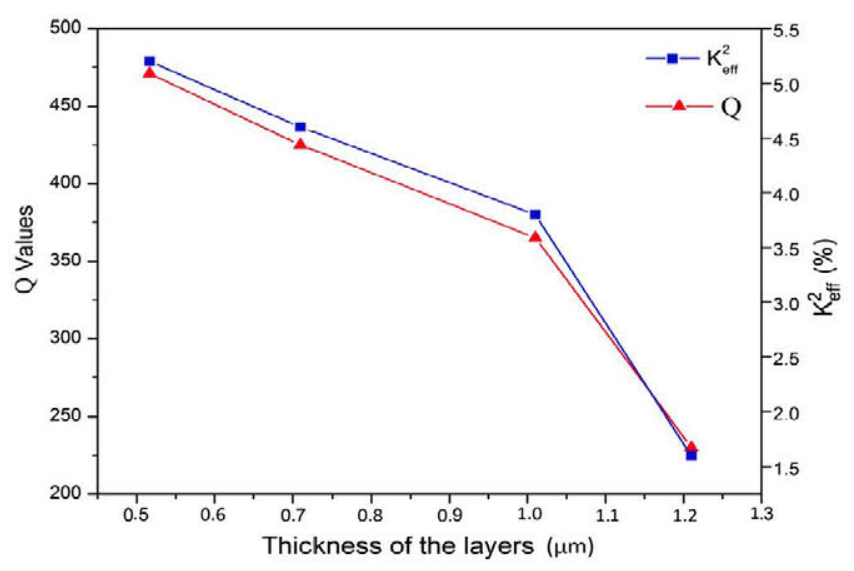

Fig. 7. Measured values of $Q$ factor and $k_{\text {eff }}^{2}$ as a function of increasing thickness of $\mathrm{SiO}_{2}$ layer in the W-based reflectors. to the generation of two overtone modes, shear and longitudinal, that appear into the $\mathrm{SiO}_{2}$ layer. This increase in the thickness of $\mathrm{SiO}_{2}$ also causes the reduction of negative temperature coefficient of frequency (Table 1). A further increase in the thickness turns the TCF for shear and longitudinal of $\mathrm{SiO}_{2}$ to positive values (in the case of $\mathrm{W}-\mathrm{SiO}_{2}$ ) based reflectors. The best possible TCF compensation is achieved when the thickness of the uppermost $\mathrm{SiO}_{2}$ layer reaches $1.21 \mu \mathrm{m}$. The further increase also reduces the TCF but causing a great degradation of the reflectance, which leads to energy leakage from the device to the substrate and a decrease in the $Q$ factor.

Similarly, to count the effect of the thickness variations of the first high impedance layer ( $\mathrm{W}$ or $\mathrm{WO}_{\mathrm{x}}$ in this case), we also increase the thickness of this layer. It is observed that by increasing the thickness of $W$ up to $0.65(\mu \mathrm{m})$, the TCF shows a decrease of the negative values compared to the symmetric configuration. Further increase in $\mathrm{W}$ thickness causes the increase in negative values of TCF shown in Fig. 8. This shows that a careful design of the resonator reducing the thickness of the uppermost $\mathrm{W}$ layer enables to decrease the TCF without a heavy layer of $\mathrm{SiO}_{2}$.

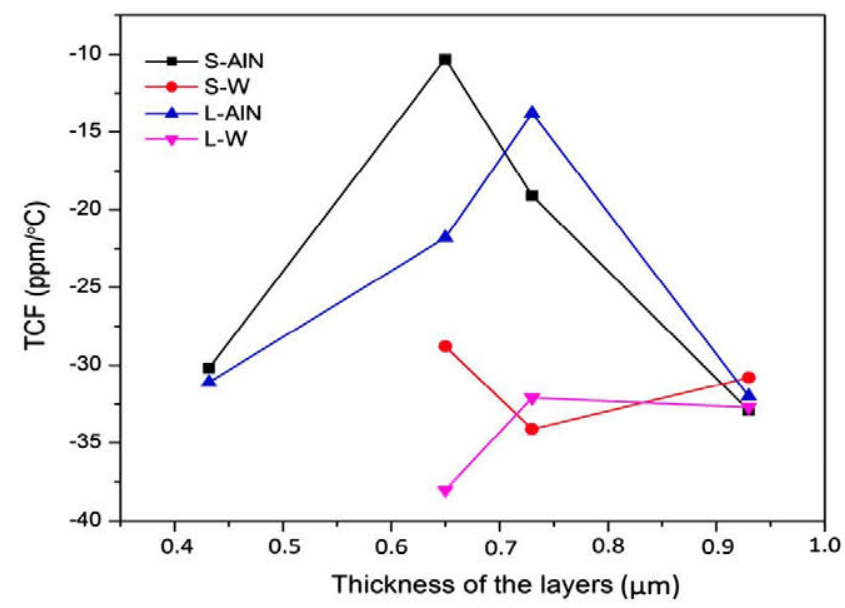

Fig. 8. Measured values of TCF of the main (AIN) and secondary (W) shear (S) and longitudinal (L) modes for asymmetric resonators as a function of the thickness of $\mathrm{W}$ in SMR reflectors.

Table 1

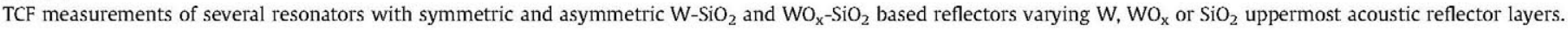

\begin{tabular}{|c|c|c|c|c|}
\hline Thickness & S-AlN & $\mathrm{S}-\mathrm{SiO}_{2}$ & L-AlN & $\mathrm{L}-\mathrm{SiO}_{2}$ \\
\hline Symmetric W-reflector & -30.2 & - & -31.1 & - \\
\hline $\begin{array}{l}\text { Asymmetric W-reflector } \\
\qquad \mathrm{SiO}_{2}=0.71 \mu \mathrm{m}\end{array}$ & -26.6 & -13.3 & -18.5 & -15.8 \\
\hline $\begin{array}{l}\text { Asymmetric W-reflector } \\
\mathrm{SiO}_{2}=1.01 \mu \mathrm{m}\end{array}$ & -24.4 & 2.6 & -10.6 & 16.4 \\
\hline $\begin{array}{l}\text { Asymmetric W-reflector } \\
\qquad \mathrm{SiO}_{2}=1.21 \mu \mathrm{m}\end{array}$ & -15.2 & 3.4 & -6.6 & 23.2 \\
\hline Symmetric $\mathrm{WO}_{\mathrm{x}}$-reflector & -23.4 & - & -19.6 & - \\
\hline $\begin{array}{l}\text { Asymmetric } \mathrm{WO}_{\mathrm{x}} \text {-reflector } \\
\mathrm{SiO}_{2}=0.85 \mu \mathrm{m}\end{array}$ & -19.9 & -18.5 & -14.6 & -12.5 \\
\hline \multirow{2}{*}{$\begin{array}{l}\text { Asymmetric } \mathrm{WO}_{\mathrm{x}} \text {-reflector } \\
\qquad \mathrm{SiO}_{2}=0.95 \mu \mathrm{m}\end{array}$} & -18.2 & -17.4 & -12.2 & -5.5 \\
\hline & S-AlN & S-W & L-AlN & $\mathrm{L}-\mathrm{W}$ \\
\hline $\begin{array}{l}\text { Asymmetric } W \text {-reflector } \\
\qquad W=0.65 \mu \mathrm{m}\end{array}$ & -10.3 & -28.8 & -21.8 & -38 \\
\hline $\begin{array}{l}\text { Asymmetric W-reflector } \\
\qquad W=0.73 \mu \mathrm{m}\end{array}$ & -19.1 & -34.1 & -13.8 & -32.1 \\
\hline \multirow{2}{*}{$\begin{array}{l}\text { Asymmetric } W \text {-reflector } \\
\qquad W=9300\end{array}$} & -32.9 & -30.8 & -32 & -32.7 \\
\hline & S-AlN & $\mathrm{S}-\mathrm{WO}_{\mathrm{x}}$ & L-AlN & $\mathrm{L}-\mathrm{WO}_{\mathrm{x}}$ \\
\hline $\begin{array}{l}\text { Asymmetric } \mathrm{WO}_{\mathrm{x}} \text {-reflector } \\
\qquad \mathrm{WO}_{\mathrm{x}}=0.95 \mu \mathrm{m}\end{array}$ & -33.4 & -46.7 & -28.4 & -30.4 \\
\hline
\end{tabular}


All the mechanical coupling acoustic reflector layers accommodate in determining the resonant frequency in a layered structure. The position of the layer in the stack matters greatly because only the first two uppermost layers show significant influence on the TCF. This is due to the low amount of acoustic energy that reaches the deeper layers. To verify this, the thickness of other layers has also been varied to see their effects on TCF but it is found that these are negligible. Only the influence of the two uppermost layers and electrodes is significant. It is also confirmed, from the transmittance response of reflectors, that more thickening of the layer of $\mathrm{SiO}_{2}$ can reduce TCF but causing excessive degradation of the response of the reflectors. Fully compensated or even positive TCF for longitudinal mode can be achieved. However, in the case of shear mode, it is very hard to obtain fully compensated TCF due to the dramatically worsening in the response of the resonator.

\section{Conclusion}

A theoretical model with experimental results for temperature compensated solidly mounted resonators is presented here. In the 2-D model, the displacement parameter for energy dissipation while increasing the thickness of different layers of the reflector is presented. The 3-D model displays the overall response of the resonator. The TCF of both the shear and longitudinal mode for different thicknesses of the uppermost layers of the acoustic reflector are calculated. The TCF of longitudinal modes can be fully compensated. The increase in the thickness of the reflector layers leads to the decrease in $Q$ value and $k_{\text {eff }}^{2}$, if no redesign of the piezoelectric capacitor is made. An increase in the number of layers does not affect the TCF (except the first two uppermost layers), but it enhances the reflector performance, and improves the response of the resonator.

\section{Acknowledgements}

This work was partially supported by the European Commission through the 7th Framework Programme by the RaptaDiag project HEALTH-304814 and by the Ministerio de Economía y Competitividad del Gobierno de España through project MAT2013-45957-R.

The authors would also like to thank for the financial support by of the Ministry of Higher Education (MOHE) Malaysia and Universiti Teknologi Malaysia (UTM) Skudai, Johor, Malaysia under Grant No. $12 \mathrm{H} 75$ and $4 \mathrm{~F} 736$.

\section{References}

[1] G. Wingqvist et al., Mass sensitivity of thin film resonator devices, in: Frequency Control Symposium, 2007 Joint with the 21st European Frequency and Time Forum. IEEE International, IEEE, 2007.

[2] G. Wingqvist, V. Yantchev, I. Katardjiev, Mass sensitivity of multilayer thin film resonant BAW sensors, Sens. Actuat. A 148 (1) (2008) 88-95.

[3] M. Clement et al., DCS Tx filters using AIN resonators with iridium electrodes, IEEE Trans. Ultrason. Ferroelectr. Freq. Control 57 (3) (2010) 518-523.

[4] D. Chen et al., Highly sensitive detection of organophosphorus pesticides by acetylcholinesterase-coated thin film bulk acoustic resonator mass-loading sensor, Biosens. Bioelectron. 41 (2013) 163-167.

[5] M. Nirschl et al., Film bulk acoustic resonators for DNA and protein detection and investigation of in vitro bacterial S-layer formation, Sens. Actuat. A 156 (1) (2009) 180-184.

[6] R. Gabl et al., Novel integrated FBAR sensors: a universal technology platform for bio-and gas-detection, in: Sensors, 2003. Proceedings of IEEE, IEEE, 2003.

[7] S. Rey-Mermet, R. Lanz, P. Muralt, Bulk acoustic wave resonator operating at 8 $\mathrm{GHz}$ for gravimetric sensing of organic films, Sens. Actuat. B: Chem. $114(2)$ (2006) 681-686.

[8] H. Zhang, E.S. Kim, Micromachined acoustic resonant mass sensor, J. Microelectromech. Syst. 14 (4) (2005) 699-706.

[9] R. Brederlow et al., Biochemical sensors based on bulk acoustic wave resonators, in: Electron Devices Meeting, 2003. IEDM'03 Technical Digest. IEEE International, IEEE, 2003.
[10] J. Weber et al., Shear mode FBARs as highly sensitive liquid biosensors, Sens. Actuat. A 128 (1) (2006) 84-88.

[11] K. Lakin et al., Temperature coefficient and ageing of BAW composite materials, in: Frequency Control Symposium and PDA Exhibition, 2001. Proceedings of the 2001 IEEE International, IEEE, 2001.

[12] M. Lakin et al., Improved bulk wave resonator coupling coefficient for wide bandwidth filters, in: Ultrasonics Symposium, 2001 IEEE. 2001, IEEE, 2001.

[13] J. Wang, K. Lakin, A. Landin, Sputtered C-Axis Inclined Piezoelectric Films and Shear-Wave Resonators, Ames Lab, IA, USA, 1983.

[14] K. Lakin, K. McCarron, J. McDonald, Temperature compensated bulk acoustic thin film resonators, in: Ultrasonics Symposium, 2000 IEEE, IEEE, 2000.

[15] J. Rosenbaum, Bulk Acoustic Wave Theory and Devices, Artech House on Demand, 1988.

[16] M. DeMiguel-Ramos et al., Assessment of the shear acoustic velocities in the different materials composing a high frequency solidly mounted resonator, Ultrasonics 62 (2015) 195-199.

[17] J. Olivares et al., Sputtered $\mathrm{SiO}_{2}$ as low acoustic impedance material for Bragg mirror fabrication in BAW resonators, IEEE Trans. Ultrason. Ferroelectr. Freq. Control 57 (1) (2010) 23-29.

[18] J. Capilla et al., High-acoustic-impedance tantalum oxide layers for insulating acoustic reflectors, IEEE Trans. Ultrason. Ferroelectr. Freq. Control 59 (3) (2012) 366-372.

[19] R. Aigner et al., Bulk-acoustic-wave filters: performance optimization and volume manufacturing, in: Microwave Symposium Digest, 2003 IEEE MTT-S International, IEEE, 2003.

[20] D. Ballantine Jr. et al., Acoustic Wave Sensors: Theory, Design, \& PhysicoChemical Applications, Academic Press, 1996.

[21] M. DeMiguel-Ramos et al., Optimized tilted c-axis AlN films for improved operation of shear mode resonators, Thin Solid Films 590 (2015) 219-223.

[22] S. Matsuda et al., Correlation between temperature coefficient of elasticity and Fourier transform infrared spectra of silicon dioxide films for surface acoustic wave devices, IEEE Trans. Ultrason. Ferroelectr. Freq. Control 58 (8) (2011) 1684-1687. 